\title{
La Productividad como factor en la mejora de la Calidad en las Unidades Médicas del IMSS Tabasco
}

\section{Productivity as a factor in the improvement of the Quality in the Medical Units of the IMSS Tabasco}

\author{
RODRIGUEZ-AYALA, Elizabeth†่, CASTRO-DE LA CRUZ, Jucelly y CUSTODIO-IZQUIERDO, \\ Carlos Arturo
}

T.M./Instituto Tecnológico de Villahermosa

ID 1 ${ }^{\text {er }}$ Autor: Elizabeth, Rodriguez-Ayala / ORC ID: 0000-00025037-4786, CVU CONACYT ID: 913057

ID $1^{\mathrm{er}}$ Coautor: Jucelly, Castro-De La Cruz / ORC ID: 0000-0002-3862-9555, Researcher ID Thomson: G-1886-2018, CVU CONACYT ID: 739319

ID $2^{\text {do }}$ Coautor: Carlos Arturo, Custodio-Izquierdo / ORC ID: 0000-0002-8639-3858, Researcher ID Thomson: G-25592018, CVU CONACYT ID: 306249

DOI: $10.35429 / J E D .2019 .20 .6 .29 .32$

Recibido: 10 de Junio, 2019; Aceptado 30 de Septiembre, 2019

\section{Resumen}

En el presente trabajo se describe el proceso del análisis de la productividad de las unidades médicas familiares del municipio del centro en la ciudad de Villahermosa Tabasco del Instituto Mexicano del Seguro Social para que con ello se pueda proponer una mejora correctiva y continua con la finalidad de obtener con ello calidad en el servicio, atacando las áreas de oportunidad que con el análisis se podrán definir. Tiene como base principal impulsar la mejora continua e innovación de las unidades administrativas del Instituto Mexicano del Seguro Social con base en las necesidades de la población y demanda de calidad en los servicios, la utilización óptima de los recursos, el reconocimiento a la participación de los trabajadores y la mejora del ambiente laboral; esto, para proyectarlas de manera ordenada a niveles competitivos de clase mundial, permitiendo a los directivos de las unidades administrativas contar con una herramienta útil de diagnóstico y mejora continua para identificar los avances y alinear sus sistemas, sus procesos hacia las necesidades y expectativas de los usuarios, identificar de cada sistema su propósito, la forma de operar, las áreas de oportunidad y decidir con su equipo de trabajo, que mejorar, como y cuando; para avanzar en la mejora continua.

Productividad, Mejora Continua, Calidad

\begin{abstract}
This paper describes the process of analyzing the productivity of the family medical units of the municipality of the center in the city of Villahermosa Tabasco of the Mexican Social Security Institute so that a corrective and continuous improvement can be proposed with the purpose of obtain with it quality in the service, attacking the areas of opportunity that with the analysis can be defined. Its main basis is to promote the continuous improvement and innovation of the administrative units of the Mexican Social Security Institute based on the needs of the population and demand for quality services, the optimal use of resources, recognition of the participation of workers and the improvement of the work environment; this, to project them in an orderly manner to competitive world-class levels, allowing the managers of the administrative units to have a useful diagnostic tool and continuous improvement to identify the advances and align their systems, their processes towards the needs and expectations of the users, identify the purpose of each system, the way they operate, the areas of opportunity and decide with their team, what to improve, how and when; to advance continuous improvement.
\end{abstract}

Productivity, Continuous Improvement, Quality

Citación: RODRIGUEZ-AYALA, Elizabeth, CASTRO-DE LA CRUZ, Jucelly y CUSTODIO-IZQUIERDO, Carlos Arturo. La Productividad como factor en la mejora de la Calidad en las Unidades Médicas del IMSS Tabasco. Revista de Desarrollo Económico. 2019. 6-20: 29-32

\footnotetext{
* Correspondencia del Autor (iscrodaya @ outlook.com)

$\dagger$ Investigador contribuyendo como primer autor.
} 


\section{Introducción}

El análisis enfocado en el aspecto de productividad incluye a todas las instituciones donde la población solicita el servicio para la mejora de su salud, así como el contexto físico, social o económico de los lugares en donde se realiza la demanda de la atención.

Teniendo en cuenta que la productividad es una medida económica que calcula cuantos bienes y servicios se han producido por cada factor utilizado durante un tiempo determinado, el aumento de esta es tan importante porque permite mejorar la calidad de vida de una sociedad.

La productividad es una palabra clave para la calidad en el servicio que el mismo exige no solo midiéndola sino insistiendo en la mejora continua para la satisfacción del usuario. La productividad está ligada a la calidad de los factores importantes en la atención de un servicio.

El servicio de atención de salud pública siempre ha sido un foco latente en la opinión del ciudadano, en la necesidad de la mejora y cubrir los requerimientos que exige la población el Instituto Mexicano del Seguro Social se ve en la necesidad de alinearse y cumplir con las demandas requeridas, el siguiente trabajo de tesis se desarrollará el análisis de la productividad de las unidades médicas familiares partiendo de ello para una propuesta de mejora, que lleva por tema "Analisis de la productividad de las unidades medicas familiares del imss del centro tabasco, para una propuesta de mejora (modelo).

Se desarrolla con la finalidad de atender los requerimientos de los usuarios (derechohabientes) en cuanto a la calidad de los servicios médicos.

\section{Antecedentes}

La tendencia actual de la sociedad tanto en el sector privado como en el público es la adopción de modelos de gestión que sirvan de referente y guía en los procesos permanentes de mejora de los productos y servicios que ofrecen. Un modelo es una descripción simplificada de una realidad que se trata de comprender, analizar y, en su caso, modificar.
Un modelo de referencia para la organización y gestión de una empresa permite establecer un enfoque y un marco de referencia objetivo, riguroso y estructurado para el diagnóstico de la organización, así como determinar las líneas de mejora continua hacia las cuales deben orientarse los esfuerzos de la organización. Es, por tanto, un referente estratégico que identifica las áreas sobre las que hay que actuar y evaluar para alcanzar la excelencia dentro de una organización.

Un modelo de gestión de calidad es un referente permanente y un instrumento eficaz en el proceso de toda organización de mejorar los productos o servicios que ofrece. El modelo favorece la comprensión de las dimensiones más relevantes de una organización, así como establece criterios de comparación con otras organizaciones y el intercambio de experiencias. De los trabajos más citados destacan los siguientes: Atkinson (1993) explora la contribución que la antropología puede hacer en la investigación de los servicios incluida la evaluación de la calidad. El conflicto entre los proveedores y los usuarios puede ser resuelto mediante la educación y el entrenamiento.

Por su parte Umar, Litaker \& Terris. (2009), al estudiar los modelos de gestión de calidad en diversos países en desarrollo concluyeron que los programas de implantación suelen estar enfocados a obtener resultados de alto impacto de corto plazo más que a la propia sustentabilidad de la calidad en el tiempo las barreras identificadas para la implantación efectiva de programas de calidad fueron dos: arranque y continuación de fases del proyecto.

Los distintos modelos de calidad que se aplican en las instituciones públicas de salud tienen diferentes enfoques y alcances. Los seis principales modelos de gestión de calidad estudiados fueron los siguientes: 1) La Secretaría de Salud, cuyo modelo que es la base para el desarrollo de otros modelos sobre todo a nivel regional y estatal. 2) La Secretaria de la Defensa Nacional, (Hospital Militar). 3) La Secretaría de Marina, (Hospital Naval de Mazatlán). 4) Petróleos Mexicanos (Pemex), (Subdirección Médica de Pemex). 5) El ISSSTE, (Instituto al Servicio de los Trabajadores al Servicio del Estado). 6) El IMSS, (Instituto Mexicano del Seguro Social). 7) Modelo NARICHA propuesto para el sistema de salud de la Escuela de Medicina y Homeopatía del Instituto Politécnico Nacional.

RODRIGUEZ-AYALA, Elizabeth, CASTRO-DE LA CRUZ, Jucelly y CUSTODIO-IZQUIERDO, Carlos Arturo. La Productividad como factor en la mejora de la Calidad en las Unidades Médicas del IMSS Tabasco. Revista de Desarrollo Económico. 2019 
El Instituto Mexicano del Seguro Social (IMSS) cuenta con un modelo de calidad que a través de los años así como ha sufrido transformaciones en su nombre también en las "bandas de madurez" que lo conforman; inicialmente se llamó a este modelo Moceri (modelo de competitividad, efectividad y resultados institucionales) actualmente es llamado MC (Modelo institucional para la competitividad) cual objetivo es obtener procesos y servicios acordes a las necesidades y expectativas de los usuarios, incrementando su satisfacción y confianza.

A pesar de que se cuenta con esta herramienta la cual sirve para mostrar resultados en los indicadores de competitividad y calidad; en si no hay una herramienta la cual ayude a reflejar indicadores de productividad.

\section{Planteamiento}

Desde los análisis de productividad que surgieron en el Instituto Mexicano del seguro social (2014) se deja entre ver la preocupación por la convergencia entre los intereses administrativos y profesionales en el ámbito de la salud ya que no suele tener compatibilidad; es por ello que se le da paso a los modelos de calidad atacando las áreas de mejora y poder solucionar el déficit en el servicio de atención principalmente en las exigencias presentadas por el usuario (derechohabiente).

\section{Justificacion}

Actualmente, existe un gran interés por la productividad en todos los ámbitos, el compromiso que se tiene por la productividad/calidad va más allá de solo hacer las cosas bien, esto incluye una serie de procedimientos realizados bajo ciertas normativas que permitan que el producto o servicio que se brinde al cliente cumpla con las exigencias que la sociedad de hoy demanda.

En cuanto a la gestión de la productividad, es la función directiva que desarrolla y aplica la política de calidad a través de cuatro procesos: planificación, organización, control de calidad y mejora continua.
La presente tesis tendrá la finalidad de analizar la productividad de las unidades médicas familiares en el municipio del centro en la ciudad de Villahermosa Tabasco al ya haber alcanzado un premio en la calidad será una buena base para atender y atacar las áreas de mejora, de igual forma esta propuesta podrá ser utilizada en cualquier área administrativa o de servicios en cualquier unidad médica.Unidades como el Hospital General de Zona No. 46 (HGZ 46), Unidad Familiar de Medicina No. 47 (UMF 47) son reconocidas por obtener el premio de Calidad, conociendo su nivel de competitividad será un buen parámetro para identificar las áreas de oportunidad en las próximas unidades a medir obteniendo resultados integrales.

\section{Objetivo general}

Generar una propuesta de mejora en las unidades administrativas del Instituto Mexicano del Seguro Social con base en la adopción de sus criterios, enfoque y estrategias institucionales; logrando la calidad deseada en base a la productividad.

\section{Objetivos especificos}

- Identificar el contexto actual de las unidades médicas de estudio

- Seleccionar los indicadores a medir para la determinación del nivel de productividad de las unidades médicas a estudiar

- Medir los indicadores seleccionadas para determinar el nivel de productividad a estudiar

- Analizar las interrelaciones entre los indicadores involucrados en el sistema

- Diseño del modelo de mejora de la productividad en las unidades medicas

\section{Delimitacion del propblema}

El problema a resolver se centra en la medición integral de la productividad en las unidades médicas del IMSS premiadas por el modelo de calidad en la Ciudad de Villahermosa, municipio del Centro en el estado de Tabasco, de Diciembre 2018 - Marzo 2020.

\section{Metas de la investigacion}

Para Marzo 2020, diseñar un modelo de mejora de la productividad de las unidades del IMSS. 
- Conocer el funcionamiento de las unidades médicas premiadas por el modelo de calidad

Identificar si el premio otorgado a las unidades médicas van acorde al nivel de productividad satisfactorio

\section{Hipotesis}

- Los premios otorgados de calidad son acorde a los niveles de productividad obtenidos

- Los factores cultural, social, económico y político inciden en los niveles de productividad en las unidades médicas.

La productividad de las unidades del IMSS se lograra incrementar a través del diseño de un modelo de mejora basado en el análisis del contexto actual.

\section{Variables}

Dependiente: Productividad

Variable independiente:

$\begin{array}{ll}- & \text { Cultural } \\ - & \text { Social } \\ - & \text { Económica } \\ - & \text { Política }\end{array}$

\section{Metodologia de la investigacion}

La investigación a desarrollar va dependiendo de los fines que se persigan, cuando el investigador descanta por algún tipo o por la combinación de más de uno.Una manera de clasificar el tipo de investigación es por el tipo de datos que se recojan; entre ellos se puede observar la cuantitativa y cualitativa o mixta.

En la presente tesis el tipo de metodología a utilizar será la mixta, esta metodología se identifica por tener características de las metodologías anteriormente mencionadas; el primer tipo de metodología (cuantitativa) se basa en ciencias empíricas centrándose en aspectos de cuantificación utilizando datos estadísticos para poder analizar los datos obtenidos; por otro lado está la metodología cualitativa que si bien no da resultados en cifras; el investigador si lo puede interpretar basándose en su observación social, son recolección de datos no estandarizados y por ende su análisis no es estadístico.
Para desarrollar el siguiente trabajo de investigación planteado primero se tiene que identificar el contexto actual que se tiene en las unidades médicas que se estudiaran; son aquellas que han obtenido el premio de calidad a nivel institucional (MC), una vez estudiadas se seleccionara los indicadores a medir para que con ello se determine el nivel de productividad de dichas unidades médicas. Dentro de los indicadores involucrados en el sistema se tendrán que analizar sus interrelaciones dando como resultado el diseño del modelo que se pretende desarrollar para la mejora de la productividad en las unidades médicas involucradas. 